\title{
Versatile Bottom-Up Approach to Nanostructured Functional Materials for Optoelectronic Applications
}

\author{
Giorgio Macchi, ${ }^{1,2}$ Francesco Meinardi, ${ }^{1,2}$ Patrizia Valsesia, ${ }^{1,3}$ and Riccardo Tubino ${ }^{1,2}$ \\ ${ }^{1}$ Dipartimento di Scienza dei Materiali, Università di Milano Bicocca, via R.Cozzi 53, 20125 Milano, Italy \\ ${ }^{2}$ Istituto Nazionale per la Fisica della Materia, Italy \\ ${ }^{3}$ Consorzio Interuniversitario per la Scienza e Tecnologia dei Materiali, Italy
}

Correspondence should be addressed to Giorgio Macchi, giorgio.macchi@mater.unimib.it

Received 28 March 2008; Accepted 8 May 2008

Recommended by Mohamed Sabry Abdel-Mottaleb

\begin{abstract}
A versatile strategy to load ordered mesoporous silica-based materials with functional molecular building blocks in order to obtain host-guest systems exhibiting specific functions is described. Optical microscope examination of the obtained material confirms that the addition of a chromophoric moiety to the reaction mixture is not detrimental in achieving the desired macroscopic morphology of the silica particles. A micro-photoluminescence investigation of the obtained material gave strong evidence that both surfactant micelles and the porous oxide matrix preserve the spectral features of the included molecular species in a nonconventional chemical environment.
\end{abstract}

Copyright (c) 2008 Giorgio Macchi et al. This is an open access article distributed under the Creative Commons Attribution License, which permits unrestricted use, distribution, and reproduction in any medium, provided the original work is properly cited.

\section{INTRODUCTION}

Since the discovery of conducting polymers, much effort has been put into the development of polymer-based optoelectronic devices (OEDs) [1-3]. Organic polymers are ideal low-cost and high-versatility materials for the rapidly evolving markets of plastic-based information and energy technologies $[4,5]$. Nevertheless, there are still problems associated with the application of organic materials in OEDs (e.g., aggregation-induced emission quenching, low quantum yield [6]). The idea described in this paper aims at overcoming some of these problems by developing photoresponsive organic/inorganic hybrid systems obtained by hierarchical organization of functional organic molecules in arrays of nanostructured host-guest compounds. Hybrid materials provide advantages which cannot be achieved with the single components. For example, the design of hybrid solar cells offers both, efficient light absorption of organic materials and efficient conversion of light to electric energy in inorganic materials $[5,7]$.

In recent years, among the great variety of porous hosts, both inorganic and organic [8-10], the synthesis of ordered mesoporous inorganic-based materials [11, 12], especially MCM-41, by employing supramolecular assembly of surfactant to template the reaction of inorganic species has been the subject of considerable research, due to their large surface area $\left(1000 \mathrm{~m}^{2} / \mathrm{g}\right)$ and storage capacity for applications in catalysis, gas, and vapours adsorption, drug delivery, molecular confinement, flow cytometry, and skincare products [13-20]. Such mesostructurally ordered porous materials have been produced in the form of micrometric powders [21], thin films [22], and bulk monoliths [23]. The key idea underlying the present paper is the addition of functional molecular building blocks to the reaction mixture, in order to obtain a host-guest material exhibiting specific functions. This bottom-up approach will lead to many advantages: (i) the oxide matrix effectively shields the guest species form environmental moisture and chemical impurities (solvents, oxygen); (ii) the proper choice of the molecular components and host geometrical constraints produces a precise control of the supramolecular organization of the inserted molecules, thus tailoring the properties of the resulting system by preventing aggregation processes even at high concentration; (iii) the obtained nanostructured material should maintain the desired macroscopic morphology. 


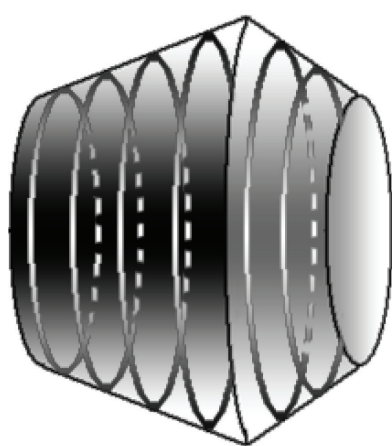

(a)

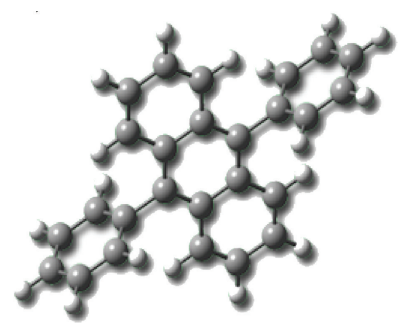

(b)
FIGURE 1: (a) Sketch of a silica particle with the expected gyroidal morphology, showing the arrangement of surfactant micelles. (b) Molecular structure of DPA.

The results that we will describe in this paper would open up the way to the preparation of active materials in various applications, such as optoelectronic devices and luminescent probes.

\section{EXPERIMENTAL}

\subsection{Synthesis of dye-loaded silica particles}

Silica-based organic-inorganic hybrid systems are obtained by a templating process: MCM-like materials were synthesized following the synthetic route described in [24]. The surfactant used was cetyltrimethylammonium chloride (CTACl, 98\%, Fluka Co., Buchs, Switzerland). Tetraethyl orthosilicate (TEOS, >99\%, from Fluka Co., Buchs, Switzerland) was the silica precursor. Oxide growth takes place in acidic conditions, and hydrochloric acid $(\mathrm{HCl})$ was consequently used. In order to prepare micrometer-sized gyroidal particles [21] (see Figure 1(a)), the following molar ratio was used: 100:4:0.11:0.13 $\mathrm{H}_{2} \mathrm{O} / \mathrm{HCl} / \mathrm{CTACl} / \mathrm{TEOS}$.

Accordingly to the literature [25], the length of CTACl alkane chains determines both micelle diameter and the pore size of the obtained material, which is of about $3.5 \mathrm{~nm}$. Under quiescent condition, cylindrical micelles arrange themselves into gyroidal shapes containing concentric channels coiling around the main axis (see Figure 1(a)).

In a typical synthesis, $355 \mathrm{mg}$ of CTACl were mixed in $15.52 \mathrm{~mL}$ of deionized water in a polypropylene bottle. The solution was kept under mild stirring at room temperature (RT) and $3.29 \mathrm{~mL}$ of $\mathrm{HCl}(37 \% \mathrm{~W}$, Fluka Co., Buchs, Switzerland) was added dropwise as well as $0.29 \mathrm{~mL}$ of TEOS. $2.06 \mathrm{mg}$ of diphenylanthracene (DPA, $\geq 98.0 \%$, Fluka Co., Buchs, Switzerland, see Figure 1(b)) was finally mixed to the mixture. After a few minutes of mild stirring, the polypropylene flask was closed and kept at RT for 3 hours. The obtained suspension was centrifugated $5000 \mathrm{rpm}$ for 5 minutes, the supernatant removed, and the resulting white powder was extensively washed with $10 \mathrm{~mL}$ of deionized water and dried in air. The white powder was then placed between a microscope slide and the cover glass for optical

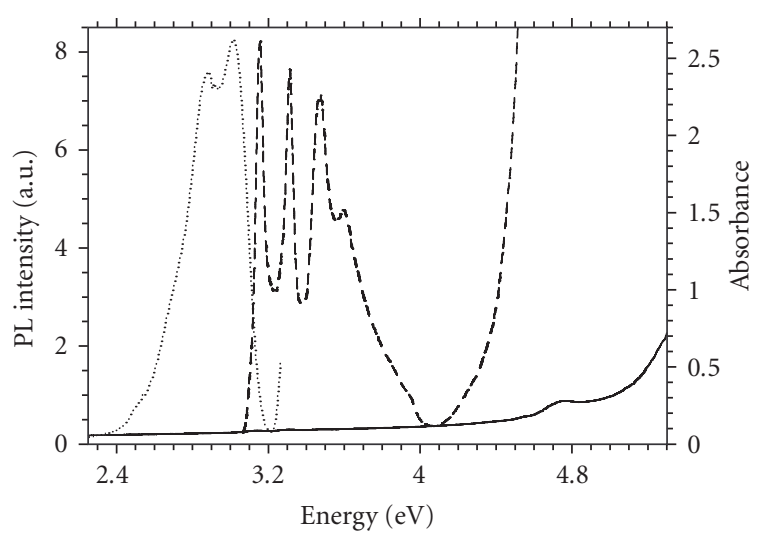

FIgURE 2: Comparison between optical absorption measurements of an aqueous solution of DPA alone (solid line) and after addition of a sample amount of CTACl (dashed line), along with PL spectrum of the resulting system (dotted line) excited at $3.15 \mathrm{eV}$.

data acquisition. A test solution of DPA in deionized water (DPA-W) with different concentration of CTACl was prepared in order to check DPA solubility dependence on the hydrophobicity of the solvent.

\subsection{Optical data acquisition}

Optical absorption and photoluminescence (PL) spectra on aqueous solutions were recorder using a Varian Cary 50 Scan UV-visible spectrophotometer (bandwidth $1 \mathrm{~nm}$ ) and a Varian Cary Eclipse spectrofluorimeter (bandwidth $1 \mathrm{~nm}$ ), respectively.

Reflected-light microimages were acquired using a doubled Nd:YAG laser (532 nm) as light source, coupled via single-mode optical fiber to an 80i Nikon confocal microscope, and a 100X oil-immersion objective (1.3 NA) to collect the light from sample. The signal is then amplified with a photomultiplier and a software-based scanning system reconstructs the overall image. Micro-PL spectra of silica particles were obtained with the same setup, using a filtered (3.26-3.76 eV bandpass) high-pressure $\mathrm{Hg}$ lamp as excitation source and by coupling the microscope to a $\mathrm{N}_{2}$-cooled CCD camera through a $190 \mathrm{~nm}$ polychromator via a multimodal optical fiber.

\section{RESULTS AND DISCUSSION}

In order to test our approach, we test DPA as a suitable molecule for the inclusion in the silica matrix by means of spectroscopic investigation of its optical features in different environments.

In Figure 2, optical absorption spectrum of a DPA-water mixture (solid line) is reported, showing no significant bands or peaks in the range $3.0-4.0 \mathrm{eV}$.

With DPA being water-insoluble, aggregates are probably formed and precipitation consequently takes place. Upon addition of surfactant (CTACl), a well-resolved vibronic progression becomes evident (see Figure 2, dashed line), with the 0-0 purely electronic transition centered at $3.15 \mathrm{eV}$. Both the shape and position of the spectrum are known in 


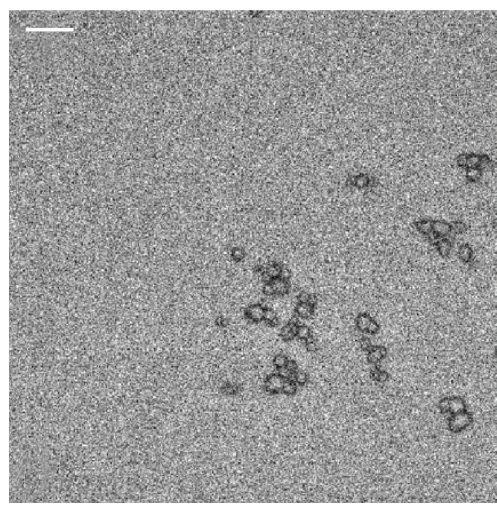

(a)

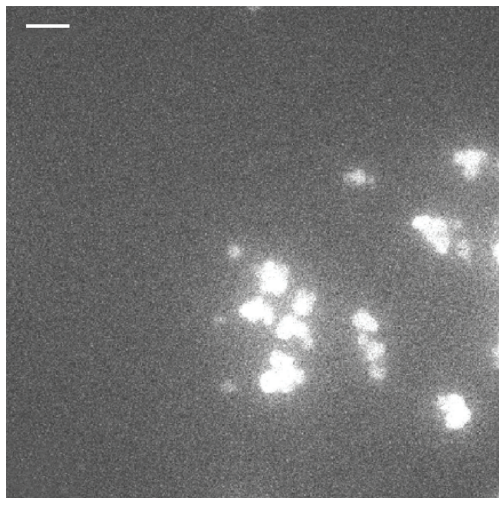

(b)

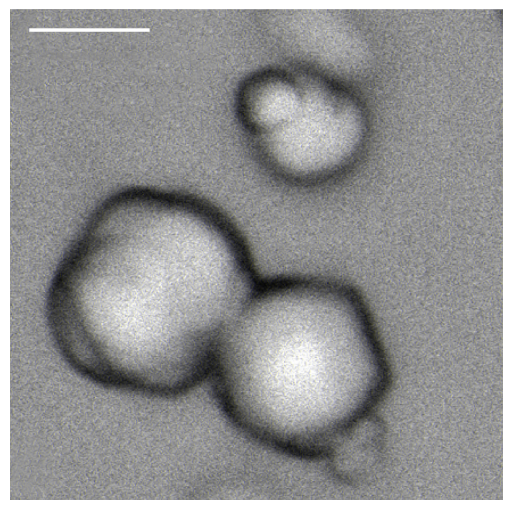

(c)

Figure 3: Microscope images of MCM-DPA sample, (a) and (b) reflected and emitted light, respectively, from silica particles on glass substrate and (c) software-assisted zoom, showing silica powder morphology (scale bars $10 \mu \mathrm{m}$ ).

literature to be fingerprint of isolated molecule behaviour [26]. PL spectrum of the DPA-water-CTACl ternary mixture is plotted in Figure 2 (dotted line), with the maximum at $2.85 \mathrm{eV}$, accordingly to the reported emission of isolated DPA in dilute solution [26]. These findings suggest that the interior of micelles formed in the water-surfactant system is able to host DPA molecules, preventing aggregation process. Moreover, following the key idea described in Section 1, silica growth should take place around such all-organic host-guest systems.

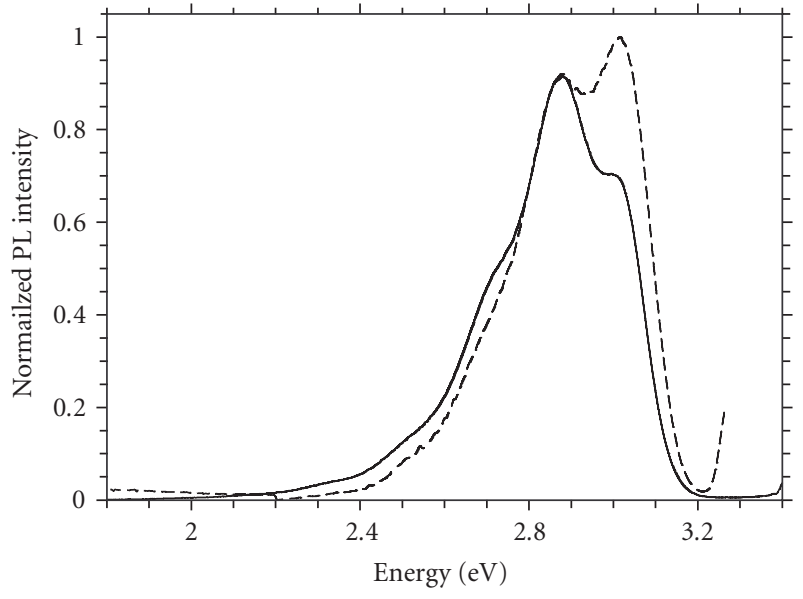

FIGURe 4: PL spectra of MCM-DPA sample (solid line) and DPAwater-CTACl ternary system (dashed line), excited with Hg lamp in the range $3.26-3.76 \mathrm{eV}$ and with $\mathrm{Xe}$ lamp at $3.15 \mathrm{eV}$, respectively.

Figure 3 reports microscope images of the sample prepared (MCM-DPA) accordingly to procedure described in Section 2.1. In Figure 3(a), a layer of silica particles dried in air shows quite good homogeneity both in shapes and in dimensions. Figure 3 (b) reports the same portion of the material under UV light, showing that emitted light is coming entirely from the silica beads. Figure 3(c) depicts a magnification of single silica particles: gyroidal shape confirms (see Figure 1(a) as reference) that the synthesis took place as expected and that the insertion of the chromophore does not affect the macroscopic morphology of the sample.

Micro-PL investigation of the particles in Figure 3(c) let us to inspect the emissive properties of the nanostructured material. The obtained PL spectrum is reported in Figure 4 (solid line) compared to emission spectrum of DPA-waterCTACl ternary system described above (dashed line). MCMDPA emission shows a structured, quite resolved band spanning from 2.0 to $3.2 \mathrm{eV}$, with a maximum centered at $2.88 \mathrm{eV}$. The vibronic progression underlying this $\mathrm{PL}$ band is quite easily recognizable, with the purely electronic transition at about $3.0 \mathrm{eV}$ and $0-1,0-2$, and $0-3$ replica at 2.7, 2.5, and $2.3 \mathrm{eV}$, respectively.

The main difference between the two spectra reported in Figure 4 concerns the intensity of the $0-0$ purely electronic transitions, being the shoulder in MCM-DPA spectrum about $70 \%$ of the corresponding peak in DPA-water-CTACl system emission. This is probably due to self-absorption phenomena, which is an evidence for the high concentration of the chromophore achievable within the silica microparticles.

\section{CONCLUSIONS}

In summary, an effective procedure to obtain hierarchicallyorganized nanostructured systems has been tested and described. The material prepared maintains the desired spectral features as well as the expected macroscopic morphology. A $\sim 30 \%$ of self-absorption observed in the silica microparticles emission spectrum demonstrates that a high 
concentration of the loaded chromophore can be achieved, producing very bright luminescent material by preventing aggregation processes (which are known to quench PL) that usually take place in more conventional systems.

These results therefore appear to be very promising for the inclusion of functional molecular and supramolecular units in inorganic host matrices as active materials in various applications, such as optoelectronic devices, luminescent probes, and hybrid solar cells.

\section{ACKNOWLEDGMENTS}

This work was supported by the European Commission through the Human Potential Programme (Marie-Curie RTN NANOMATCH, Grant no. MRTN-CT-2006-035884). R.Tubino thanks Fondazione Cariplo and FIRB Synergy for the financial support. P.Valsesia also thanks FIRB Idee Progettuali.

\section{REFERENCES}

[1] M. D. McGehee and A. J. Heeger, "Semiconducting (conjugated) polymers as materials for solid-state lasers," Advanced Materials, vol. 12, no. 22, pp. 1655-1668, 2000.

[2] Z. Bao, "Materials and fabrication needs for low-cost organic transistor circuits," Advanced Materials, vol. 12, no. 3, pp. 227230, 2000.

[3] R. H. Friend, R. W. Gymer, A. B. Holmes, et al., "Electroluminescence in conjugated polymers," Nature, vol. 397, no. 6715, pp. 121-128, 1999.

[4] T. W. Kelley, P. F. Baude, C. Gerlach, et al., "Recent progress in organic electronics: materials, devices, and processes," Chemistry of Materials, vol. 16, no. 23, pp. 4413-4422, 2004.

[5] S. E. Shaheen, C. J. Brabec, N. S. Sariciftci, F. Padinger, T. Fromherz, and J. C. Hummelen, " $2.5 \%$ efficient organic plastic solar cells," Applied Physics Letters, vol. 78, no. 6, pp. 841-843, 2001.

[6] D. Oelkrug, A. Tompert, J. Gierschner, et al., "Tuning of fluorescence in films and nanoparticles of oligophenylenevinylenes," Journal of Physical Chemistry B, vol. 102, no. 11, pp. 1902-1907, 1998.

[7] W. U. Huynh, J. J. Dittmer, and A. P. Alivisatos, "Hybrid nanorod-polymer solar cells," Science, vol. 295, no. 5564, pp. 2425-2427, 2002.

[8] G. Calzaferri, H. Maas, M. Pauchard, M. Pfenniger, S. Megelski, and A. Devaux, "Supramolecularly organized luminescent dye molecules in the channels of zeolite L" in Advances in Photochemistry, Vol. 27, pp. 1-50, John Wiley \& Sons, New York, NY, USA, 2002.

[9] G. Bongiovanni, C. Botta, G. Di Silvestro, M. A. Loi, A. Mura, and R. Tubino, "Energy transfer in nanostructured oligothiophene inclusion compounds," Chemical Physics Letters, vol. 345, no. 5-6, pp. 386-394, 2001.

[10] B. D. Wagner, "Recent applications of host-guest inclusion in fluorescence-based trace analysis," Current Analytical Chemistry, vol. 3, no. 3, pp. 183-195, 2007.

[11] A. Corma, "From microporous to mesoporous molecular sieve materials and their use in catalysis," Chemical Reviews, vol. 97, no. 6, pp. 2373-2420, 1997.

[12] Q. Huo, D. I. Margolese, U. Ciesla, et al., "Organization of organic molecules with inorganic molecular species into nanocomposite biphase arrays," Chemistry of Materials, vol. 6, no. 8, pp. 1176-1191, 1994.
[13] D. E. De Vos, M. Dams, B. F. Sels, and P. A. Jacobs, "Ordered mesoporous and microporous molecular sieves functionalized with transition metal complexes as catalysts for selective organic transformations," Chemical Reviews, vol. 102, no. 10, pp. 3615-3640, 2002.

[14] H. Yoshitake, T. Yokoi, and T. Tatsumi, "Adsorption of chromate and arsenate by amino-functionalized MCM-41 and SBA-1," Chemistry of Materials, vol. 14, no. 11, pp. 4603-4610, 2002.

[15] M. Vallet-Regí, A. Rámila, R. P. del Real, and J. PérezPariente, "A new property of MCM-41: drug delivery system," Chemistry of Materials, vol. 13, no. 2, pp. 308-311, 2001.

[16] P. Sozzani, S. Bracco, A. Comotti, et al., "Complete shape retention in the transformation of silica to polymer microobjects," Nature Materials, vol. 5, no. 7, pp. 545-551, 2006.

[17] M. Wark, Y. Rohlfing, Y. Altindag, and H. Wellmann, "Optical gas sensing by semiconductor nanoparticles or organic dye molecules hosted in the pores of mesoporous siliceous MCM41," Physical Chemistry Chemical Physics, vol. 5, no. 23, pp. 5188-5194, 2003.

[18] B. J. Scott, G. Wirnsberger, and G. D. Stucky, "Mesoporous and mesostructured materials for optical applications," Chemistry of Materials, vol. 13, no. 10, pp. 3140-3150, 2001.

[19] M. Choi, F. Kleitz, D. Liu, H. Y. Lee, W.-S. Ahn, and R. Ryoo, "Controlled polymerization in mesoporous silica toward the design of organic-inorganic composite nanoporous materials," Journal of the American Chemical Society, vol. 127, no. 6, pp. 1924-1932, 2005.

[20] S. Iyer, Y. Kievsky, and I. Sokolov, "Fluorescent silica colloids for study and visualization of skin care products," Skin Research \& Technology, vol. 13, no. 3, pp. 317-322, 2007.

[21] H. Yang, N. Coombs, and G. A. Ozin, "Morphogenesis of shapes and surface patterns in mesoporous silica," Nature, vol. 386, no. 6626, pp. 692-695, 1997.

[22] L. Huang, S. Kawi, K. Hidajat, and S. C. Ng, "Formation of mesoporous silica thin films on oxide substrates by casting," Microporous and Mesoporous Materials, vol. 88, no. 1-3, pp. 254-265, 2006.

[23] N. A. Melosh, P. Davidson, and B. F. Chmelka, "Monolithic mesophase silica with large ordering domains," Journal of the American Chemical Society, vol. 122, no. 5, pp. 823-829, 2000.

[24] T. Itoh, K. Yano, Y. Inada, and Y. Fukushima, "Photostabilized chlorophyll $a$ in mesoporous silica: adsorption properties and photoreduction activity of chlorophyll $a$," Journal of the American Chemical Society, vol. 124, no. 45, pp. 13437-13441, 2002.

[25] I. Sokolov, Y. Y. Kievsky, and J. M. Kaszpurenko, "Selfassembly of ultrabright fluorescent silica particles," Small, vol. 3, no. 3, pp. 419-423, 2007.

[26] Y. Hirshberg, "The absorption spectra of phenylated anthracenes," Transactions of the Faraday Society, vol. 44, pp. 285-289, 1948. 


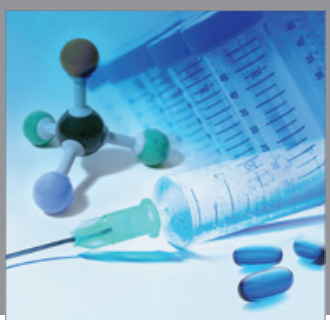

International Journal of

Medicinal Chemistry

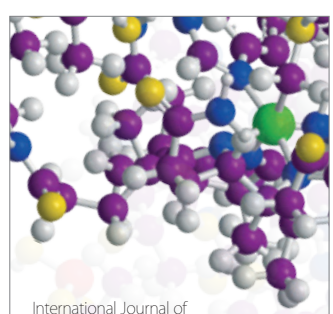

Carbohydrate Chemistry

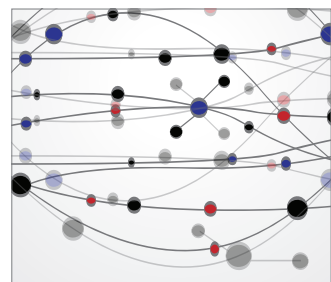

The Scientific World Journal
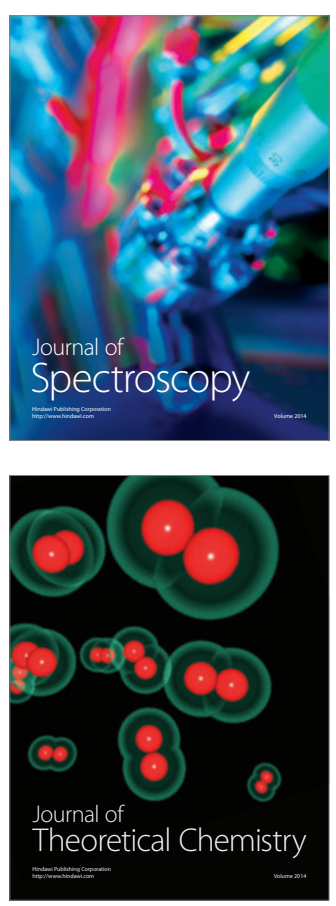
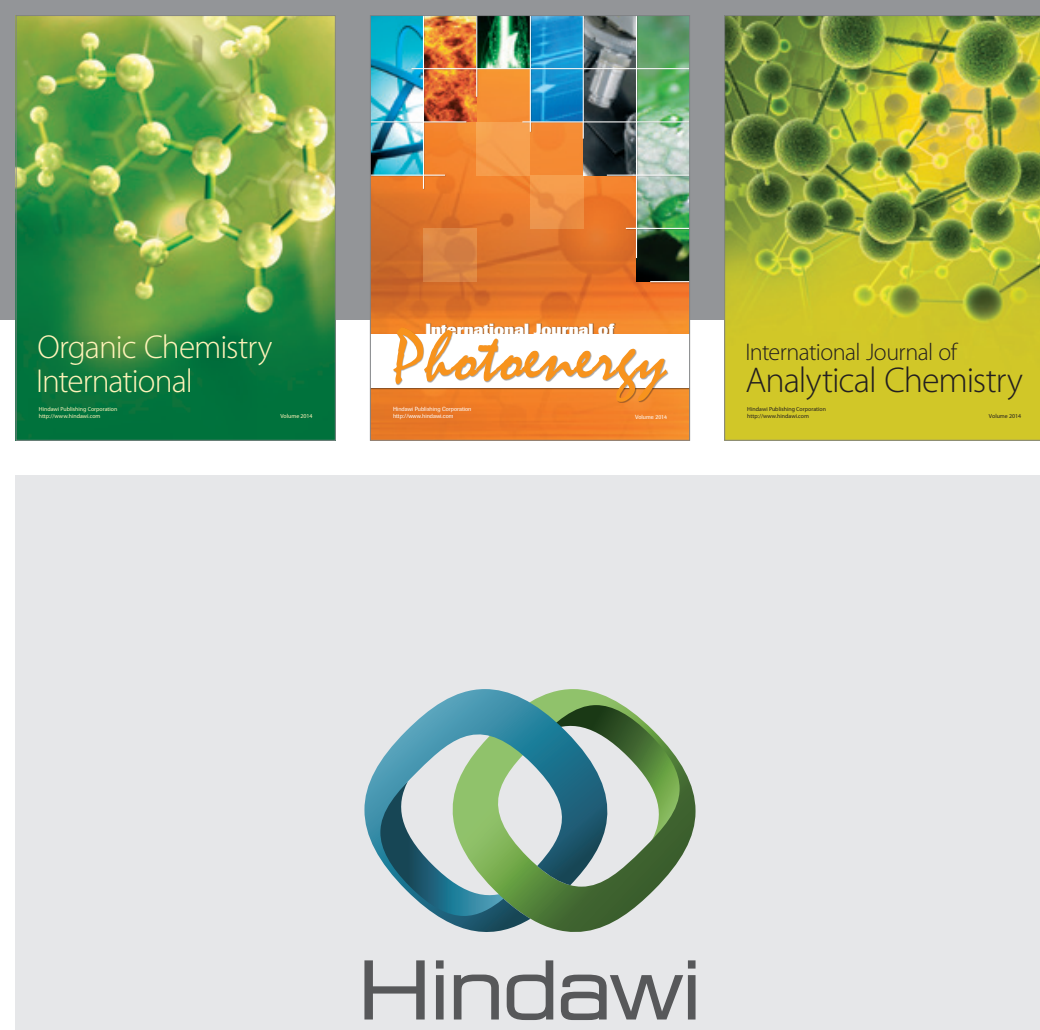

Submit your manuscripts at

http://www.hindawi.com
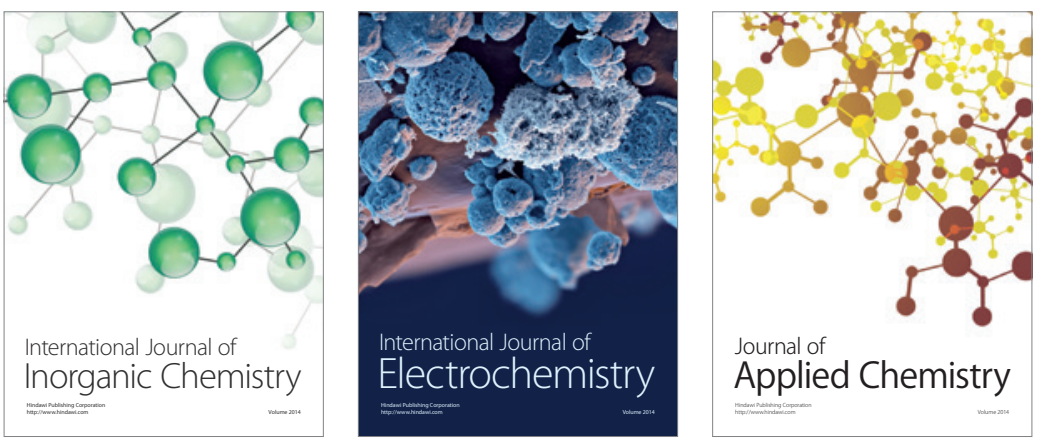

Journal of

Applied Chemistry
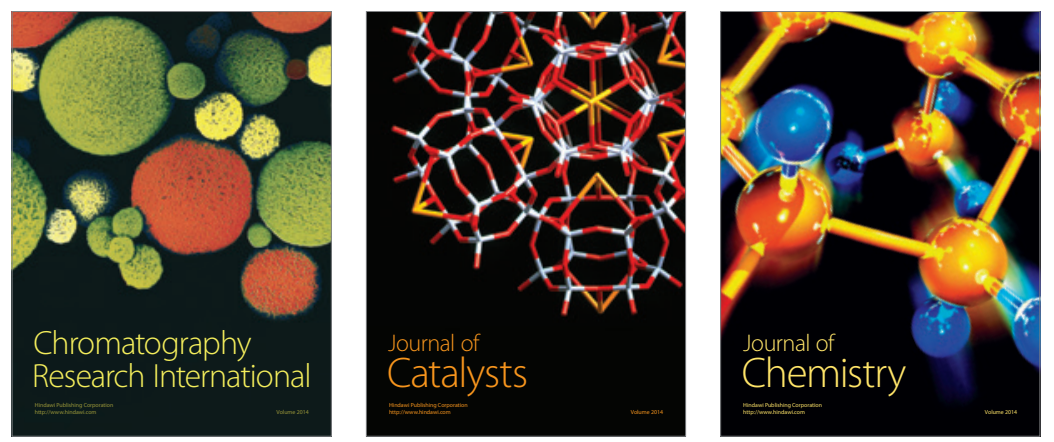
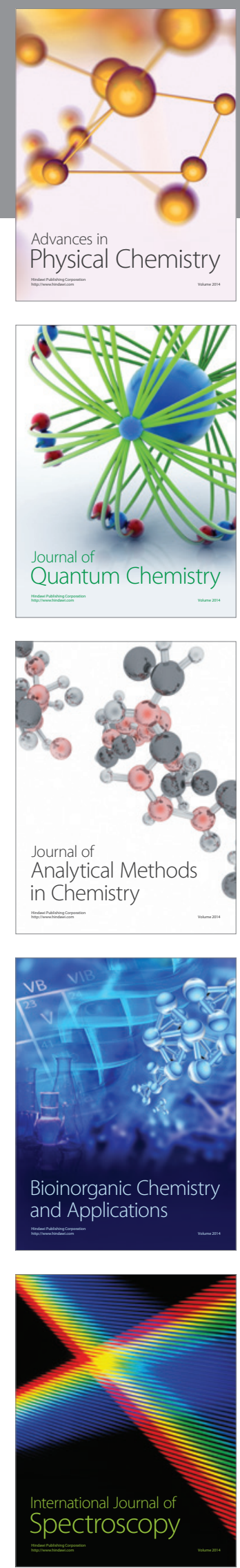